\title{
Optimal Design of EMALS Based on a Double Sided Tubular Linear Induction Motor
}

\author{
Antonino Musolino, Marco Raugi, Rocco Rizzo, and Mauro Tucci \\ DESTEC, Department of Engineering for System Energy, University of Pisa, Italy
}

\begin{abstract}
A semi-analytical model of the Double Sided Tubular Linear Induction Motor (DSTLIM) is adopted for the optimization of the thrust force of the stages of an ElectroMagnetic Aircraft Launch System (EMALS). The model allows a fast and accurate prediction of all the electromagnetic quantities in the device, including the thrust force, the back-electromotive force, the distribution of the induced current and the average magnetic flux density in the teeth. This provides a basis for the design which has been performed by a novel evolutionary optimization algorithm. By using the semi-analytical formulation, the characterization of the machine is greatly facilitated so allowing a fast evaluation of the cost function and of the design constraints.
\end{abstract}

\section{INTRODUCTION}

The Electro-Magnetic Aircraft Launch System (EMALS) is intensely investigated as it seems to be able to overcome the limits of steam catapults to launch aircraft from carriers. The thermodynamics of the steam cycle poses an upper bounds on the performance of the steam catapult, in particular on the maximum launch energy which could hardly exceed 100MJ. This value is still appropriate for the weight of actual aircraft but it could not be sufficient for those of the next generation [1], [2]. The steam catapult is a conglomerate of heterogeneous subsystems: hydraulic, electromechanical, thermal, each with associated motors and control systems, complicating the launch process.

The most important drawback of steam catapults is the lack of feedback control. Thrust force is affected by large transients that can damage or reduce the life of the airframe. Moreover due to the unpredictability of the thrust force, extra force (often unnecessary) is added. The peak to mean acceleration ratio is nominally 1.25 (with excursion up to 2.0 ).

EMALS is a fully integrated system consisting of an energy storage system, a power electronics system, a linear motor, and a control system. Whit EMALS the same linear motor is used to launch, brake and retract the shuttle, eliminating a number of auxiliary components and simplifying the overall system. The energy is kinetically stored in rotors spinning at $6400 \mathrm{rpm}$ taking energy from the ship's electric distribution and making it available for pulse discharge during the launch. The pulse discharge for proper feeding of the linear motor is conditioned by the power electronics system. The control system supervises all the launch activities according to specified parameters.

TABLE I. EMALS REQUIREMENTS

\begin{tabular}{|l|l|}
\hline end speed & $28 \div 103 \mathrm{~m} / \mathrm{s}$ \\
\hline max peak-to-mean force ratio & 1.05 \\
\hline launch energy & $122 \mathrm{MJ}$ \\
\hline cycle time & $45 \mathrm{~s}$ \\
\hline maximum braking distance & $10 \mathrm{~m}$ \\
\hline maximum thrust & $1.3 \mathrm{MN}$ \\
\hline maximum length & $\approx 100 \mathrm{~m}$ \\
\hline
\end{tabular}

The main requirements of an aircraft launch system are summarized in table I.

The choice of the linear motor is a central issue in the EMALS design since its feeding conditions determine the design and the operation of both the control power conditioning systems.

The two candidates are the Linear Permanent Magnet Synchronous Motor (LPMSM) and the Linear Induction Motor (LIM). Both are "long stator" machine with a wound segmented stator spanning the entire acceleration path. The moving shuttle consist of an array of permanent magnet for LPMSM, or of a conductive plate for LIM. The double sided topology is usually adopted in both cases.

When LPMSM are used the switching frequency of the drive electronics must be, at a minimum, in the range of 1800-4200 $\mathrm{Hz}$. These frequencies may border the limits of the capabilities of high-power drives based on IGBT or IGCT. LIM are able to provide the same thrust force with voltages and currents of approximately the same magnitude but with fundamental frequencies in the range of $30-250 \mathrm{~Hz}$ [3].

Because of their highest force for moving mass ratio, Tubular Linear Induction Machines (TLIMs) [4-10] are very attractive for use in the EMALS context, especially with regard to the braking requirements.

Standard TLIMs are characterized by a single sided configuration; in a previous paper the authors have proposed a Double Sided TLIM (DSTLIM) consisting of two concentric stators, with the mover between them [11].

A semi-analytical model of the DSTLIM has been developed in [11], and a good accuracy has been established by comparison 
with numerical FEM models. In this paper the semi-analytical model is used to the design optimization of a multistage EMALS. This approach has revealed to be effective, while a numerical approach may result in unviable computation times.

The optimization procedure here adopted is based on an original evolutionary algorithm for global optimization, proposed by the authors in [12]. This approach is based on the paradigm of competitive neural networks and Self-Organizing Maps (SOM) [13], and its performance has shown to be comparable with the state-of-the-art of evolutionary optimization algorithms [12]. The proposed approach tries to take advantage of the SOM strong explorative power, by defining a new optimization strategy where the SOM centroids represent individuals that move in the search space looking for low fitness values.

The paper is organized as follows: Section II discusses the EMALS based on the DSTLIM; section III describes the design optimization procedure and finally Sect. IV presents the optimization results.

\section{THE EMALS MOTOR}

\section{A. The proposed configuration}

The DSTLIM proposed for the use in the EMALS is deeply discussed in [11]. For the sake of readability a 3D view of it is depicted in Fig. 1, while Fig. 2 shows a cross section in correspondence of the middle of a tooth.

When a multistage configuration is adopted, all the stages share the same mover and the same distance between the stators. Each stage of the proposed configuration consists of four symmetrically positioned DSTLIM as shown in Fig. 3 [14].

This configuration presents some noteworthy advantages with respect to the use of a single DSTLIM with the same cross section:

a) the surface of the active portion of the mover is greater. For example let us consider four $2 m$ long DSTLIMs with a mean radius of the mover of $25 \mathrm{~cm}$ and an outer stator thickness of 15 $\mathrm{cm}$ (Fig. 2). The total active portion of the mover is

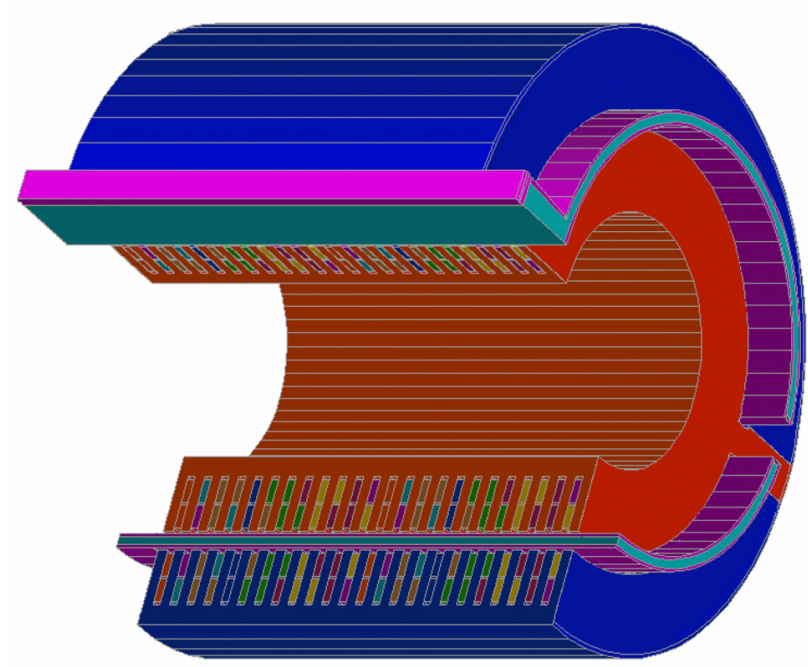

Fig. 1. Schematic 3D view of the DSTLIM (courtesy of [9]).

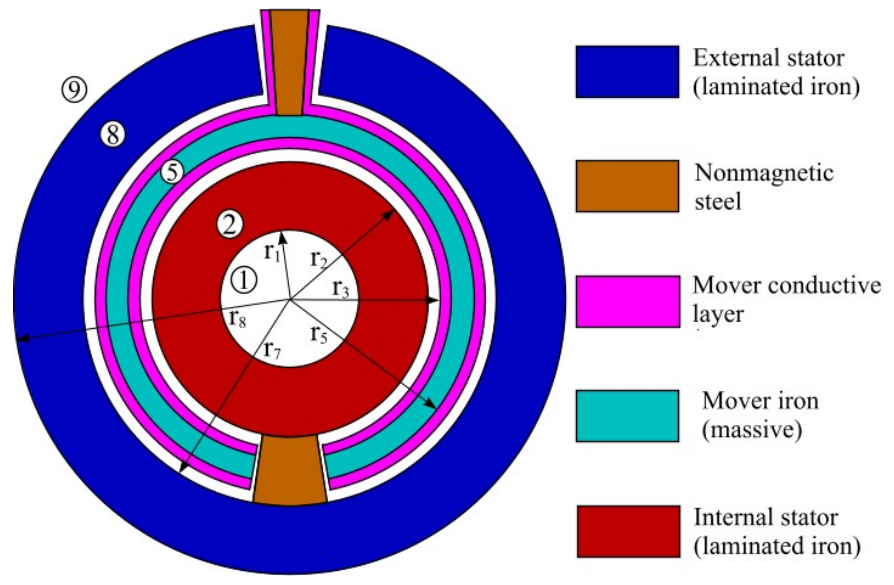

Fig. 2. A view of the cross section of the DSTLIM (courtesy of [9]).

$2 \times 4 \times 2 \pi \times 0.25 \times 2=25.14 \mathrm{~m}^{2}$. Assuming a separation between the four DSTLIMs of $10 \mathrm{~cm}$, the side of the square containing the entire arrangement is $170 \mathrm{~cm}$. The active surface of the mover if a single DSTLIM with a radius of $70 \mathrm{~cm}$ (assuming the same thickness of the external stator) were used would $2 \times 2 \pi \times 0.7 \times 2=17.56 \mathrm{~m}^{2}$.

b) The feeding voltages of each smaller DSTLIM is a fraction of the feeding voltage of the bigger one. Assuming the same feeding current, the corresponding voltage is proportional to the radius. Smaller voltages are easier to produce and to control. 


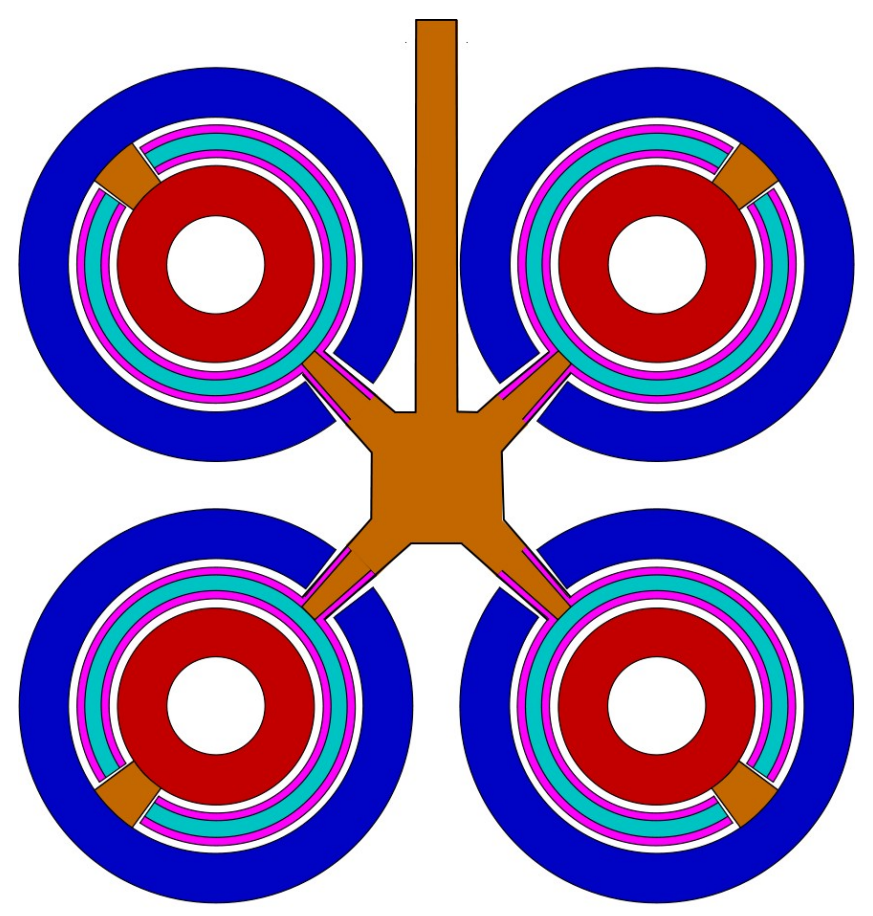

Fig. 3. Schematic representation of the cross section of the EMALS motor.

Moreover, since the motors are asynchronous, there is no need of synchronization between the voltages that may be generated by independent systems.

c) There is an intrinsic redundancy in the system. If a failure occurs in one of the DSTLIMs (caused by the motor itself, the feeding, or the control systems) the other modules can be overloaded in order to compensate for the decrease of the thrust force.

The drawbacks related to the proposed configuration are the increased complexity of the structure, and the need of multiple feeding and control systems. However they are of smaller size than those needed by a single module configuration.

\section{B. $\quad$ The feeding strategy}

As known, when the speed of the mover is allowed to vary in a large range, a multistage design is commonly adopted in order to improve the overall efficiency. The stator is segmented in several sections which are fed sequentially. In our design we consider 50 stages of equal length of $2 \mathrm{~m}$. The windings on both the stators of each stage are distributed in 48 slots, two coils per slot, to produce a travelling wave of flux density in the airgap characterized by four pole pairs. We assumed a total length of the mover of $6 \mathrm{~m}$; the mean radius of the moving part of each of the four DSTLIMs is $19 \mathrm{~cm}$. The thickness of the central iron layer is $15 \mathrm{~mm}$, and the thickness of the inner and outer conductive layer (aluminum) are $6.75 \mathrm{~mm}$ and $11.25 \mathrm{~mm}$ respectively. The total weight of the mover is about $1770 \mathrm{~kg}$ (442 $\mathrm{kg}$ per component). Both the airgaps between the mover and the stators are $7.5 \mathrm{~mm}$.

Fig. 4 shows the feeding condition of the stages as a function of the mover position. The stator stages are schematically indicated by the colored rectangles, while the mover is indicated by the gray rectangle. A stator winding is fed when the mover has entirely entered the corresponding section. In Fig. 4 the red color indicates fed sections that completely overlap with the mover, light blue indicates sections which are not fed, and orange is used for the sections which are fed but

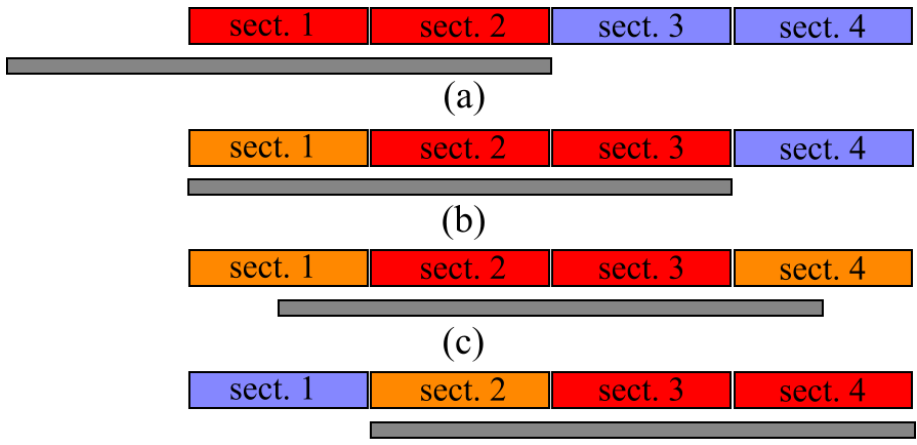

(d)

Fig. 4. Feeding the sections of the stators.

partially overlap with the mover. The contribution of the orange stages to the total thrust force is expected to be a fraction of the thrust force due to a red portion. Authors in [15] introduce a coefficient $K_{p}=l_{o v} / L_{s e c}$ where $l_{o v}$ is the portion of the mover that overlaps with the section of the stator and $L_{s e c}$ is the length of the section. In this paper we consider the action of the red sections only, discarding the contribution of the orange ones, so allowing $K_{p}$ to assume the values 0 and 1 only; this produces underestimated values of the thrust force.

\section{OPTIMIZATION PROCEDURE}

An optimization with respect to the geometrical parameters and the feeding condition has been performed by an evolutionary, global optimization algorithm proposed by the authors, and denoted by the acronym Soc-opt ( Self organizing centroids optimization) [12]. This stochastic and population based algorithm is based on a modified version of the SOM and competitive neural networks [16], [17]. The goal is to minimize a real parameter objective function, $F(\mathbf{x})\left\{F: \Omega \subseteq \Re^{D} \rightarrow \Re\right\}$, $\mathbf{x} \in \Omega$, where $\Omega$ is the domain of the search.

\section{A. Optimization algorithm description}

The proposed algorithm creates a two dimensional grid of $P$ cells where a distance metric $d(c, i)$ is defined between each 
pair of cells of index $c, i=1 \ldots P$. A centroid vector $\mathbf{c}^{i}(t) \in \mathfrak{R}^{D}$, and a local best solution $\mathbf{t}^{i}(t) \in \mathfrak{R}^{D}$ are associated to each cell. A global best solution $\mathbf{t}^{\text {ghest }}(t) \in \mathfrak{R}^{D}$ is defined as the minimum $\mathbf{t}^{i}(t) \in \Re^{D} \quad i=1 \ldots P$. The time index $t=0,1,2 \ldots T_{\max }$ represents the iteration index and $T_{\max }$ is the maximum number of iterations. Random initialization is considered. The value of the fitness function $F\left(\mathbf{t}^{i}(t)\right)$ in the local best solutions is known, and the cell searches in the input space around the centroid $\mathbf{c}^{i}(t)$ for a possible better vector $\mathbf{t}^{i}(t)$. The centroid is perturbed according to the following (equivalent to mutation):

$\mathbf{p}^{i}(t)=\mathbf{c}^{i}(t-1)+\boldsymbol{\delta}^{i}(t-1)$,

in which $\boldsymbol{\delta}^{i}(t-1) \in \mathfrak{R}^{D}$ is a random perturbation, whose components are uniformly distributed in $\left[-d_{\text {neigh }}, d_{\text {neigh }}\right]$, where $d_{n e i g h}=\left\|\mathbf{c}^{i}-\mathbf{c}^{\text {neigh(i) }}\right\|$, and neigh(i) is a reference neighbour cell of the cell $i$.

The fitness value is calculated according to $F\left(\mathbf{p}^{i}(t)\right)$ in the points that verify the given constraints. The local best solutions are the updated according to:

$\mathbf{t}^{i}(t)=\left\{\begin{array}{ll}\mathbf{p}^{i}(t) & \text { if } F\left(\mathbf{p}^{i}(t)\right) \leq F\left(\mathbf{t}^{i}(t-1)\right) \\ \mathbf{t}^{i}(t-1) & \text { otherwise }\end{array}\right.$,

which is similar to the selection step in evolutionary optimization algorithms. The winning cell is defined as, gbest $=\arg \min \left(F\left(\mathbf{t}^{i}(t)\right)\right)$. As a result of the selection made in

(15), $F\left(\mathbf{t}^{\text {gbest }}(t)\right)$ corresponds to the global best fitness value found in all generations.

At this point each centroid $\mathbf{c}^{i}(t)$ moves in the direction of a convex combination of its solution vector $\mathbf{t}^{i}(t)$ and the global best solution $\mathbf{t}^{\text {ghest }}(t) \in \Re^{D}$, which is given by the following input vector:

$\mathbf{x}^{i}(t)=\lambda^{i}(t) \mathbf{t}^{\text {gbest }}(t)+\left[1-\lambda^{i}(t)\right] \mathbf{t}^{i}(t)$,

where $\lambda^{i}(t)=\exp \left(-d(\text { gbest }, i)^{2} / 2 \sigma_{\lambda}^{2}\right)$ is a neighbourhood

function as in the classical SOM algorithm, $\lambda^{i}(t) \in(0,1]$

The movement of the centroid $\mathbf{c}^{i}(t)$ towards the input $\mathbf{x}^{i}(t)$ is implemented by a linear, time invariant, discrete time filter. As a consequence the centroid vector tracks the input vector following a given dynamic, which depends on the choice of the filter of order $N$, which has the following transfer function:
$G(z)=\frac{b_{1} z^{N-1}+\ldots+b_{N}}{z^{N}-a_{1} z^{N-1}-a_{2} z^{N-2} \ldots-a_{N}}$.

The transfer function (17) is defined using the Z-Transform formalism, and the coefficients $\left[b_{1}, \ldots, b_{N}\right]$ and $\left[a_{1}, \ldots, a_{N}\right]$ are fixed and define the particular filtering action between the input sequence $\mathbf{x}^{i}(t)$ and output sequence $\mathbf{c}^{i}(t)$. To track the past values of the input and output sequences the following memory vectors are introduced: $\left[\mathbf{x}_{1}^{i}, \ldots, \mathbf{x}_{N}^{i}\right],\left[\mathbf{c}_{1}^{i}, \ldots, \mathbf{c}_{N}^{i}\right]$, where $\mathbf{c}_{j}^{i} \in \Re^{D}, \mathbf{x}_{j}^{i} \in \Re^{D}, i=1 \ldots P, j=1 \ldots N$. At each iteration, the memory vectors are updated as follows, for $j=1 \ldots N, i=1 \ldots P$

$$
\left\{\begin{array}{l}
\left\{\begin{array}{l}
\mathbf{x}_{0}^{i}(t-1)=\mathbf{x}^{i}(t), \mathbf{c}_{0}^{i}(t-1)=\mathbf{c}^{i}(t-1) \\
\mathbf{x}_{j}^{i}(t)=\mathbf{x}_{j}^{i}(t-1)+\lambda^{i}(t)\left(\mathbf{x}_{j-1}^{i}(t-1)-\mathbf{x}_{j}^{i}(t-1)\right) . \\
\mathbf{c}_{j}^{i}(t)=\mathbf{c}_{j}^{i}(t-1)+\lambda^{i}(t)\left(\mathbf{c}_{j-1}^{i}(t-1)-\mathbf{c}_{j}^{i}(t-1)\right)
\end{array}\right.
\end{array}\right.
$$

which performs an intermediate one step time shift of the memory vectors taking into account the neighbourhood collaboration (only the memories of cells near the winning one are substantially updated).

The new centroid vector, which moves towards the input, is finally evaluated by:

$\mathbf{c}^{i}(t)=\sum_{j=1}^{N} b_{j} \mathbf{x}_{j}^{i}(t)+a_{j} \mathbf{c}_{j}^{i}(t)$,

which represents an implementation of the transfer function (17). When the centroid tracks the input $\mathbf{x}^{i}(t)$ it automatically tracks the best local solutions $\mathbf{t}^{i}(t)$, and it is also attracted towards the global best solution $\mathbf{t}^{\text {ghest }}(t)$, depending on the value of $\lambda^{i}(t)$. Centroids in the cells near to the gbest $\left(\lambda^{i}(t) \approx 1\right)$ are mainly attracted by the global best. After the centroid update in (19), a new perturbation step (14) is performed.

At the end of the optimization, which is reached when a maximum number of function evaluations have been performed, we can say that the targets $\mathbf{t}^{i}\left(T_{\max }\right)$ represent the final candidate solutions and $\mathbf{t}^{\text {gbest }}\left(T_{\max }\right)$ is the global best solution found by the algorithm. The proposed algorithm exhibits a good behaviour, when compared to well known evolutionary optimization algorithms [12].

\section{B. Constrains handling}

A set of direct constraints is introduced by defining maximum and minimum allowed values for each component of the solution vector, which represent an optimization variable. If a component of the randomly perturbed vector $\mathbf{p}^{i}(t)$ obtained in (14) does not respect the constraints, it is substituted by a randomly 
generated value that lies within the allowed interval before the evaluation of the objective function, $F\left(\mathbf{p}^{i}(t)\right), i=1 \ldots P$.

Indirect constraints are also defined on quantities which have a complex dependence on the optimization variables. In order to prevent iron saturation, we introduce a maximum allowed magnetic flux density. In the same fashion the maximum induced currents on the conductive layers of the mover are constrained, in order to limit the overheating. These constrained quantities, magnetic flux and current densities, are calculated, and the vectors $\mathbf{p}^{i}(t)$ are separated in two groups. The first group contains the vectors that respect all the indirect constraints, while the second group contains the others. If the first group is empty the optimization process is stopped. Otherwise each vector of the second group is discarded and substituted with a vector randomly selected from the first group. Constraints handling is carried out before the selection step (15); in this way best local solution vectors $\mathbf{t}^{i}(t)$, which are the candidate solutions, always verify both direct and indirect constraints.

\section{APPLICATION EXAMPLES}

Optimization procedures require several (typically thousands) evaluations of the objective function. The use of numerical models to describe the device, especially at speed, results in unviable computation times.

Equivalent circuit models may represent a valid tool which allows saving times while retaining a good accuracy [18]. Circuit models not always are able to describe the distribution of electromagnetic quantities inside the motor. The approach based on the electromagnetic analysis described in [11] allows more accurate description of the device while retaining acceptable computation times.

In this section we consider an example of optimization of the stator sections of the multistage DSTLIM as described in Sect. II. The relative permeability of the laminated iron for the stators is 1000 and its conductivity is zero; massive iron in the central layer of the mover has conductivity $\sigma_{f e}=1 \cdot 10^{7} \mathrm{~S} / \mathrm{m}$, with the same relative permeability as before, while the conductive layers of the mover (aluminum) have $\sigma_{A l}=3.7 \cdot 10^{7} \mathrm{~S} / \mathrm{m}$. Other assigned quantity are: the mean radius of the mover ( $\left.r_{m, f e}=19 \mathrm{~cm}\right)$, the thickness of the central iron layer ( $\left.\delta_{m, f e}=15 \mathrm{~mm}\right)$ and the thickness of the inner $\left(\delta_{A l, i}=6.75 \mathrm{~mm}\right)$ and outer $\left(\delta_{A l, e}=11.25 \mathrm{~mm}\right)$ conductive layer.

We look for the maximum thrust force on the mover for a given current density ( $\left.J_{\text {stat }}=11 \mathrm{~A} / \mathrm{mm}^{2}\right)$ in the stator coils with respect to geometric and feeding parameters. The search space is constituted by the slots dimensions (width and depth) of both stators and by the feeding frequency.

The direct constraints (those set on the parameters that are in the search space) are: $0.25 \cdot \tau_{\text {slot }}<\Delta z_{\text {slot int }}<0.75 \cdot \tau_{\text {slot }}$, $30<\Delta r_{\text {slot, int }}<80$, $0.25 \cdot \tau_{\text {slot }}<\Delta z_{\text {slot, ext }}<0.75 \cdot \tau_{\text {slot }}$ $50<\Delta r_{\text {slot,ext }}<150, \Delta z$ is the slot width, $\Delta r$ the slot depth and $\tau_{\text {slot }}$ is the slot pitch. Dimension are expressed in $\mathrm{mm}$.

As indirect constraints we assign the maximum values on the current density in the conductive parts of the mover $J_{\text {mov }}=45 \mathrm{~A} / \mathrm{mm}^{2}$ (rms value) and the maximum value of the average flux density in the teeth where the flux density is expected to assume its maximum value $B_{\text {iooih }}=2.2 T$ (peak value). The constraint on the current produces an admissible temperature rise on the mover conductive layer as predicted by the Onderdonk's formula [19]. As far the constraint on the magnetic flux density, this high value is allowed because it is usually reached only in limited portions of some teeth.

There are always (at least) two stages fed at a time, as described in Sect. II b). A total of 8 stator sections are active in the whole system. Considering that the required thrust force is $1.3 M N$, each section has to the exert on the mover a force of $162 k N$.

Table II reports the results of the optimization for a number of selected sections. The results for the missing sections are very similar (except for the frequency) to those of neighboring sections. In addition to optimized parameters, the Table reports the achievable thrust force and the speed of the mover. In the sections where the thrust force exceeds the required value, the feeding currents have to be properly reduced.

Fig. 5 shows the distribution of the induced current density in the middle of the external conductive layer of the mover in for three values of the speed: $10 \mathrm{~m} / \mathrm{s}, 40 \mathrm{~m} / \mathrm{s}$ and $79 \mathrm{~m} / \mathrm{s}$, corresponding respectively to section numbers 2,9 and 32 . The reference of the z-axis of Fig. 5 is placed on the midpoint of the section. As expected [20], at low speed the current distribution is well localized in correspondence of the fed section. As the speed increases the current density extends outside of the fed sections in the direction of the motion.

Fig. 6 shows the distribution of the magnetic flux density in the middle on the outer airgap. The behavior is similar to that of the current density. 
TABLE II. OPTIMIZATION RESULTS

\begin{tabular}{|r|r|r|r|r|r|r|r|}
\hline $\begin{array}{r}\text { Sect. } \\
\text { Numb. }\end{array}$ & $\begin{array}{c}\text { Speed } \\
(\mathrm{m} / \mathrm{s})\end{array}$ & $\begin{array}{c}\Delta z_{\text {slot, int }} \\
(\mathrm{mm})\end{array}$ & $\begin{array}{c}\Delta r_{\text {slot, int }} \\
(\mathrm{mm})\end{array}$ & $\begin{array}{c}\Delta z_{\text {slo, ext }} \\
(\mathrm{mm})\end{array}$ & $\begin{array}{c}\Delta r_{\text {slot, ext }} \\
(\mathrm{mm})\end{array}$ & $\begin{array}{c}\text { Freq. } \\
H z\end{array}$ & $\begin{array}{c}\text { Force } \\
\mathrm{kN}\end{array}$ \\
\hline 1 & 7.07 & 19.34 & 68.04 & 21.13 & 112.42 & 16.8 & 418.3 \\
\hline 2 & 10.00 & 20.94 & 69.01 & 23.35 & 108.82 & 23.9 & 391.8 \\
\hline 3 & 19.32 & 23.98 & 72.39 & 22.79 & 117.19 & 45.6 & 316.8 \\
\hline 4 & 24.14 & 24.48 & 73.04 & 23.80 & 113.52 & 56.5 & 289.3 \\
\hline 5 & 28.06 & 25.21 & 73.10 & 24.78 & 110.09 & 65.6 & 271.0 \\
\hline 6 & 31.46 & 24.64 & 76.57 & 26.87 & 101.84 & 73.5 & 256.4 \\
\hline 7 & 34.52 & 25.24 & 76.06 & 26.59 & 103.28 & 80.5 & 245.2 \\
\hline 8 & 37.32 & 25.34 & 76.53 & 26.58 & 103.48 & 87.0 & 235.7 \\
\hline 9 & 39.92 & 25.44 & 77.17 & 26.51 & 103.96 & 93.0 & 227.7 \\
\hline 10 & 42.36 & 25.58 & 77.36 & 26.57 & 103.82 & 98.6 & 220.8 \\
\hline 11 & 44.67 & 24.91 & 62.92 & 23.32 & 103.97 & 99.5 & 178.6 \\
\hline 14 & 50.95 & 24.51 & 65.36 & 23.49 & 104.20 & 113.4 & 173.1 \\
\hline 17 & 56.54 & 24.55 & 66.12 & 23.60 & 104.31 & 125.8 & 168.4 \\
\hline 20 & 61.62 & 24.53 & 66.90 & 23.70 & 104.35 & 137.0 & 164.6 \\
\hline 23 & 66.32 & 24.65 & 66.92 & 23.74 & 104.35 & 147.3 & 161.3 \\
\hline 26 & 70.70 & 24.82 & 66.92 & 23.77 & 104.39 & 157.0 & 158.3 \\
\hline 29 & 74.82 & 23.45 & 66.30 & 23.73 & 102.99 & 158.4 & 161.4 \\
\hline 32 & 78.73 & 23.43 & 67.31 & 23.95 & 102.60 & 166.7 & 162.0 \\
\hline 35 & 82.45 & 23.39 & 67.85 & 24.03 & 102.36 & 174.5 & 162.2 \\
\hline 38 & 86.02 & 23.40 & 68.14 & 24.03 & 102.34 & 182.0 & 162.3 \\
\hline 41 & 89.44 & 23.42 & 68.60 & 24.07 & 102.44 & 189.2 & 162.5 \\
\hline 44 & 92.73 & 23.46 & 68.91 & 24.08 & 102.59 & 196.2 & 162.6 \\
\hline 47 & 95.91 & 23.59 & 68.99 & 24.07 & 102.81 & 202.9 & 162.6 \\
\hline 50 & 98.99 & 23.61 & 69.05 & 24.06 & 102.76 & 209.3 & 162.7 \\
\hline & & & & & & & \\
\hline
\end{tabular}

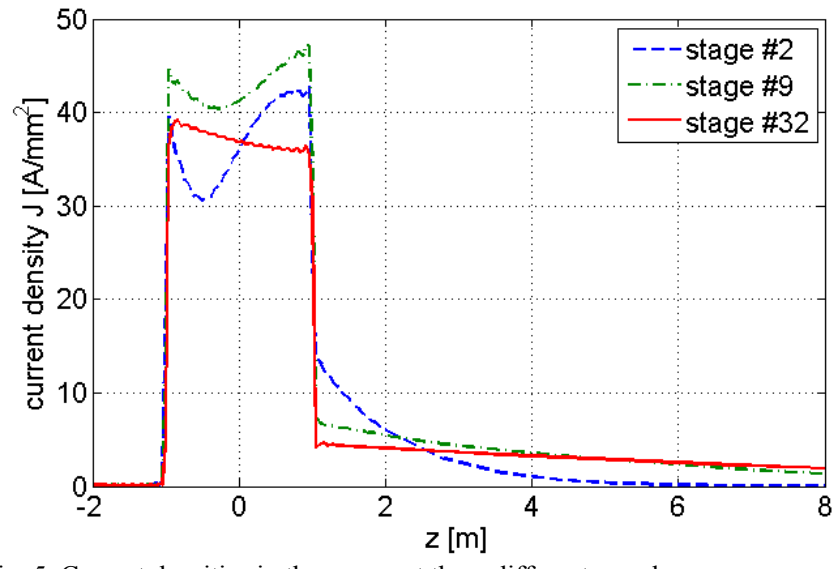

Fig. 5. Current densities in the mover at three different speeds

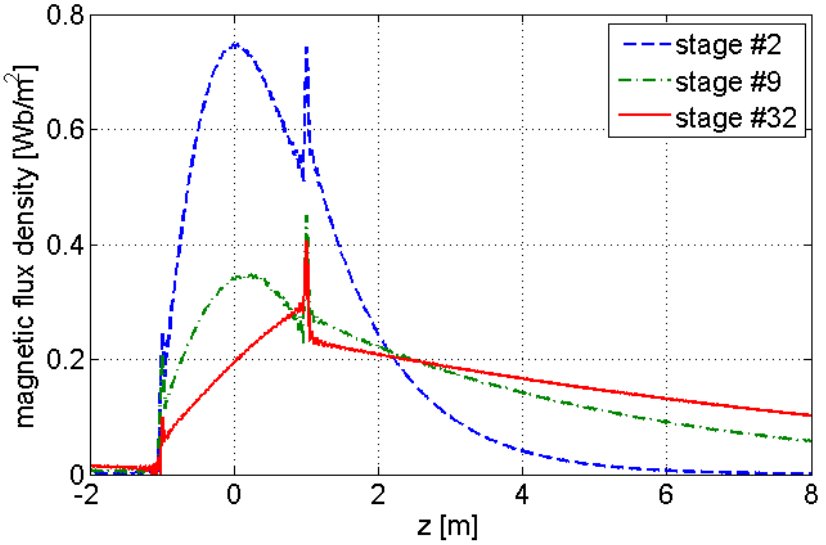

Fig. 6. Magnetic flux densities in the mover at three different speeds

\section{CONCLUSION}

A preliminary design of an EMALS based on the DSTLIM has been presented in the paper. The proposed configuration has been discussed its main characteristics have been shown. A optimization algorithm in conjunction with a semi-analytical model of the DSTLIM has been adopted to maximize the thrust force of every section. The obtained results shows the feasibility of the design.

\section{REFERENCES}

[1] M. R. Doyle, D. J. Samuel, T. Conway, and R. R. Klimowski, "Electromagnetic aircraft launch system-EMALS," IEEE Trans. Magn., vol. 31, no. 1, pp.:528 -533, Jan. 1995.

[2] R. R. Bushway, "Electromagnetic aircraft launch system development considerations.", IEEE Trans. Magn., vol. 37, no. 1, pp.: 52 -54, Jan. 2001.

[3] D. C. Meeker and M. J. Newman, "Indirect vector control of a redundant linear induction motor for aircraft launch," Proc. Of the IEEE, vol. 97, n. 11, pp. 1768-1776, Nov. 2009.

[4] V. Di Dio, G. Cipriani, R. Miceli, and R. Rizzo, "Design criteria of tubular linear induction motors and generators: a prototype realization and its characterization," Leonardo Electronic Journal of Practices and Technologies, vol. 12, pp. 23-41, May 2013.

[5] V. Di Dio, V. Franzitta, F. Muzio, G. Scaccianoce, and M. Trapanese, "The use of sea waves for generation of electrical energy and hydrogen,"in Proc. MTS/IEEE Biloxi-Marine Technol. Our Future, Global Local Challenges (OCEANS), Biloxi, MS, USA, Oct. 2009, pp. 1-4.

[6] V. Di Dio, and M. Montana, "State of the art of tubular linear induction motor," Proceeding of the Mediterranean Electrotechnical Conference, MELECON, Bari, 13-16 May 1996, pp. 285, 288.

[7] A. Musolino, R. Rizzo, and E. Tripodi, "Tubular linear induction machine as a fast actuator: analysis and design criteria," Progress in Electromagnetic Research, vol. 132, pp. 603-619, Oct. 2012.

[8] S. Barmada, A. Musolino, R. Rizzo, A. Tellini, "Fields analysis in axisymmetric actuators", IEEE Trans. Mag., vol. 36 n. 4, pp. 1906-1909, July 2000.

[9] A. Musolino, R. Rizzo, and E. Tripodi,, "Travelling Wave Multipole Field Electromagnetic Launcher: An SOVP Analytical Model," IEEE Trans Plasma Sci., vol. 41, no. 5, pp. 1201-1208, May 2013.

[10] M. Caruso, G. Cipriani, V. Di Dio, R. Miceli, C. Nevoloso, and G. D. Galluzzo, "Performance comparison of tubular linear induction motors with different primary winding connections," in Proc. ICEM, Berlin, Germany, Sep. 2014, pp. 1370-1375. 
[11] A. Musolino, R. Rizzo, and E. Tripodi, "The double sided tubular linear induction motor and its possible use in the electro-magnetic aircraft launch system," IEEE Trans Plasma Sci., vol. 41, no. 5, pp. 1193-1200, May 2013.

[12] S. Barmada, M. Raugi, and M. Tucci, "Global optimization algorithm based on self-organizing centroids," Evolutionary Computation (CEC), 2012 IEEE Congress on, pp.1-6, Brisbane, AUS, 10-15 June 2012

[13] T. Kohonen, Self-Organizing Maps, Third Edition. Springer, Berlin, 2001.

[14] U. Hasirci, A. Balikci, Z. Zabar, and L. Birenbaum, "Concerning the Design of a Novel Electromagnetic Launcher for Earth-to-Orbit Micro-and Nanosatellite Systems," IEEE Trans Plasma Sci, vol. 39, no. 1, pp.: 498503, Jan. 2011.

[15] D. Patterson, A. Monti, C. W. Brice, R. A. Dougal, R. O. Pettus, S. Dhulipala, D. C. Kovuri, and T. Bertoncelli, "Design and simulation of a permanent-magnet electromagnetic aircraft launcher," IEEE Trans. Ind. Appl., vol. 41, no. 2, pp. 566-574, Mar./Apr. 2005.

[16] M. Tucci, and M. Raugi, "Adaptive FIR Neural Model for Centroid Learning in Self-Organizing Maps," IEEE Trans. Neural. Networks., vol. 21, no. 6, pp. 948-960, Jun. 2010.

[17] M. Tucci, and M. Raugi, "A filter based neuron model for adaptive incremental learning of self-organizing maps," Neurocomputing, vol. 74, no. 11, pp. 1815-1822, May 2011.

[18] A. Shiri, and A. Shoulaie, "Design Optimization and Analysis of SingleSided Linear Induction Motor, Considering All Phenomena," IEEE Trans. on Energy Conversion, vol. 27, no. 2, pp. 516-525, Jun. 2012.

[19] D. G. Fink, and H. W. Beaty, Standard Handbook for Electrical Engineers, 12th edition, McGraw-Hill, 1999, pp. 4-74.

[20] S. A. Nasar, and I. Boldea, Linear motion electric machines, pp. 63-84, John Wiley \& Sons, New York, 1976 